\title{
Ginkgo biloba Extract Reduces Hippocampus Inflammatory Responses, Improves Cardiac Functions And Depressive Behaviors In A Heart Failure Mouse Model
}

This article was published in the following Dove Press journal:

Neuropsychiatric Disease and Treatment

\author{
Lijun Zhang' \\ Jianyang Liu' \\ Yingbin $\mathrm{Ge}^{2}$ \\ Meiyan Liu' \\ 'Department of Cardiology, Beijing \\ Anzhen Hospital Affiliated to Capital \\ Medical University, Beijing 100029 , \\ People's Republic of China; ${ }^{2}$ Department \\ of Physiology, Nanjing Medical University, \\ Nanjing, Jiangsu 21 I I66, People's \\ Republic of China
}

Correspondence: Meiyan Liu Department of Cardiology, Beijing Anzhen Hospital Affiliated to Capital Medical University, Beijing 100029 , People's Republic of China Email liumeiyanaz@I63.com
Background: Depression has been shown to share an extremely high comorbidity with heart failure (HF). Ginkgo biloba extract (GBE) is a widely used traditional Chinese medicine in cardiac disease. However, its potential therapeutic effect on depressive symptoms following HF largely remains unknown. In this article, we aimed to investigate its effects in reducing depressive behaviors of a HF mouse model. Moreover, we also discussed whether its effects are associated with changes in neural inflammation and 5-hydroxytryptamine (5-HT) signaling.

Methods: Mice were randomly divided into three groups: sham, $\mathrm{HF}+$ saline and $\mathrm{HF}+\mathrm{GBE}$ $(150 \mathrm{mg} / \mathrm{kg} / \mathrm{d})$ ( $\mathrm{n}=10$ per group). Systolic heart failure was induced by ligating the left anterior descending coronary artery. Cardiac functions together with depressive-like behaviors were measured after 4 weeks' treatment. Levels of brain natriuretic peptide (BNP), 5 HT, 5-HT receptor $2 \mathrm{~A}\left(5-\mathrm{HT}_{2 \mathrm{~A}} \mathrm{R}\right)$, tumor necrosis factor- $\alpha$ (TNF- $\left.\alpha\right)$, interleukin-1 $\beta$ (IL-1 $\left.\beta\right)$, vascular endothelial growth factor (VEGF), hypoxia inducible factor-1 (HIF-1), (cleaved) caspase-3, Bax and Bcl-2 were analyzed by Western blot, Elisa and immunohistochemistry at the end of the experiments.

Results: GBE benefited antidepressant-like behaviors and improved cardiac functions in mice with heart failure. Levels of TNF- $\alpha$, IL- $1 \beta$ and 5-HT were reduced in the hippocampus after the administration of GBE. Further experiments revealed that GBE also blocked the release of serotonin in the peripheral blood and triggered HIF-1 induced anti-apoptotic pathways.

Conclusion: GBE has potential therapeutic effects in relieving depressive status of patients with HF.

Keywords: heart failure, inflammation, 5-HT, apoptosis, depression

\section{Introduction}

Heart failure (HF) is usually regarded as the end stage of various diseases. The population suffering from HF has reached 26 million with an economic burden exceeding 31 billion USD globally. ${ }^{1}$ However, it is disappointing to note that, although advances have been achieved in recent years, the prevalence of HF is still increasing, especially in developed countries. ${ }^{2}$ Depression is a common mental illness that affects up to $60 \%$ of patients with $\mathrm{HF}$ and results in significantly worsened prognosis. $^{3-5}$ It is now widely accepted that improvements in psychological health are crucial to HF patients. Thereby, understanding the underlying 
mechanisms of this comorbidity and the potential pharmacological effects of clinically adopted drugs will be of great importance in improving long-term prognosis in patients with both HF and depression.

Recently, several studies from our own and other research groups have reported depressive behaviors in HF rodents after a myocardial infarction (MI) procedure. ${ }^{6-8}$ Although the exact mechanism still remains obscure, it may be associated with immune and 5-hydroxytryptamine (5-HT) systems. Dysfunctions in neuroendocrine systems, particularly in hypothalamic-pituitary-adrenal (HPA) axis, are now considered to be major contributors to the development of psychocardiological diseases, a classification which describes comorbidity of cardiovascular diseases and mental illness. ${ }^{9}$ The over-activation of the HPA axis, as characterized by increased levels of cortisol, always indicates elevated inflammatory and serotonergic responses, both of which play important pathological roles in psychocardiological diseases. ${ }^{8,10-12}$

Ginkgo biloba extract (GBE) is a natural product which has been widely used in treating multiple diseases, including angina pectoris, ${ }^{13}$ glaucoma, ${ }^{14}$ brain injury ${ }^{15}$ and depression. ${ }^{16}$ Its anti-apoptotic, anti-oxidative and anti-inflammatory properties are well acknowledged. ${ }^{17}$ However, few studies have focused on the therapeutic effects of GBE in this post-HF depression model. Herein, we describe how we validated depressive behaviors and explored the effects of GBE administration in HF mice. Moreover, we also investigated the involvement of neural inflammation, 5-HT signaling and cardiac apoptotic pathways in GBE's action.

\section{Materials And Methods}

\section{Animals}

Male C57BL/6 mice aged 8-10 weeks old, weighing 20-25g, were obtained from the Comparative Medicine Centre of Yangzhou University. Mice were housed at 23 $\pm 2^{\circ} \mathrm{C}$ with a 12 -hr light-dark cycle. Food and water were available ad libitum. All experiments were performed according to the NIH guide for the care and use of laboratory animals and were approved by the ethics committee of Beijing Anzhen Hospital affiliated to Capital Medical University (No. 2014016X).

Mice were randomly divided into three groups including sham + saline, $\mathrm{HF}+$ saline and $\mathrm{HF}+\mathrm{GBE}$ group $(\mathrm{N}=10$ in each group). HF was induced by ligating the left anterior descending (LAD) coronary artery as described before. ${ }^{8}$ Two weeks after the ligation surgery, left ventricular ejection fraction (LVEF) values were detected by echocardiography using the Vevo 2100 ultrasound system (Visual Sonic, Canada) (30MHz ultrasonic probe). Mice with LVEF values less than $30 \%$ were selected and assigned to HF groups. Mice in the sham group received same procedure except for LAD ligation. No animals died as a result of the surgery. After the surgery, mice in the saline groups were given $2.5 \mathrm{~mL}$ saline per day while mice in the $\mathrm{HF}+\mathrm{GBE}$ group received Ginkgo biloba dripping pills treatment $(150 \mathrm{mg} / \mathrm{kg} /$ day) (provided by WanBangDe Pharmaceutical Group Co., Ltd.). For a human, the regular dose of GBE (dripping pills) is $900 \mathrm{mg} /$ day (60mg/one pill, 5 pills/once, three times a day), therefore, we chose the drug dose of $150 \mathrm{mg} / \mathrm{kg} /$ day for a mouse whose drug dose is 10-20 times compared with a human. The pharmacological administration was performed via intragastric gavage at 7:00 a.m. every day for 4 consecutive weeks.

At the end of the experiments (after echocardiography and behavior tests), mice were anesthetized with pentobarbital and sacrificed by decapitating with a guillotine. Blood, cerebral cortex, hippocampus and cardiac tissue were collected for further analysis.

\section{Behavior Tests}

Sucrose preference tests (SPT) and open field tests (OFT) were used to describe depressive behaviors. We chose SPT and OPT due to such reasons: firstly, SPT and OPT are two standard tests for evaluating depressive behaviors as forced swim test and tail suspension test. Secondly, the laboratory limitations in our lab. In the SPT, mice were habituated to $0.5 \%$ saccharine for $24 \mathrm{hrs}$, one bottle of sucrose water and one bottle of tap water were placed on each side of the cage, and the sequence was changed after 12 hrs. ${ }^{18}$ After habituation, mice were provided with ad libitum access to sweetened water and tap water for another 24 hrs. Then, the water pouches were weighed and recorded. The sucrose preference was calculated by dividing consumption of sweetened water by total consumption of water. The experimental apparatus used in the OFT was a square black box $(60 \mathrm{~cm} \times 60 \mathrm{~cm} \times 25 \mathrm{~cm})$, made out of plexiglass with $5 \times 5$ squares. The mice were individually placed in the center of the enclosure and allowed to explore freely for 6 mins in a quiet environment. The numbers of squares crossed were recorded during the last 4 mins.

\section{Masson's Staining}

Freshly isolated hearts were fixed in paraformaldehyde (PFA) solution. After sectioning (4- $\mu \mathrm{m}$-thick), embedding 
and staining, images were observed by microscopy and were imported to the analysis software (Image J). The myocardial fibrosis area and normal myocardial area were then analyzed to evaluate myocardial fibrosis grade.

\section{Enzyme-Linked Immunosorbent Assay (ELISA)}

Platelet-rich plasma and platelets were prepared as described before. ${ }^{8}$ BNP levels in the serum, together with 5-HT and 5-HT ${ }_{2 \mathrm{~A}} \mathrm{R}$ (\#YFXEM00760; \#YFXEM00037; \# YFXEM00761, YIFEIXUE BIOTECH, Nanjing, People's Republic of China) levels in the serum, platelet, hippocampus and cortex tissues were determined using ELISA kits according to the manufacturer's protocol.

\section{Western Blotting}

Tissue was homogenized, lysed and centrifuged at $2000 \mathrm{rpm}$ for 20 mins. The supernatants were collected and protein concentrations were measured using BAC kits (Keygen Biotech, Nanjing, People's Republic of China). Proteins were separated through $10 \%$ sodium dodecyl sulfate-polyacrylamide gel electrophoresis (SDS-PAGE) (25 $\mu \mathrm{g} /$ lane), and membranes prepared by Western blot procedure were finally analyzed using the ECL system (Millipore, Bedford, USA). Primary antibodies were used as follows:

GAPDH (\#8884, 1:10000, Cell Signaling Technology, Boston USA), Caspase-3 (\#9662, 1: 1000, Cell Signaling Technology, Boston USA), Cleaved Caspase-3 (\#9664, 1:1000, Cell Signaling Technology, Boston USA), Bax (\#5023, 1:1000, Cell Signaling Technology, Boston USA), Bcl-2 (\#15071, 1:1000, Cell Signaling Technology, Boston USA), HIF-1 $\alpha$ (\#36169, 1:1000, Cell Signaling Technology, Boston USA), VEGF (ab52917, 1:1000, Abcam, England), TNF alpha (ab1793, 1:1000, Abcam, England), IL-1 beta (ab9722, 1:2000, Abcam, England).

\section{Immunohistochemistry}

Hippocampus tissue was collected and fixed in 4\% PFA. After embedding and sectioning (4-um-thick), slides were deparaffinized and rehydrated. Antigen retrieval was performed with the citrate buffer $(\mathrm{pH}$ 6.0) by heating the slides for 20 mins using a microwave oven. After blocking endogenous peroxidase by $3 \% \mathrm{H} 2 \mathrm{O} 2$, sections were incubated overnight at $4^{\circ} \mathrm{C}$ with the goat polyclonal anti-TNF alpha antibody (ab1793, 1:50, Abcam, England) or anti-IL-1 beta antibody (ab9722, 1:1000, Abcam, England) at $4^{\circ} \mathrm{C}$. Twostep technique (SuperPictureTM3rd Gen IHC Detection kit;
Invitrogen, CA, USA) was used for visualization, with diaminobenzidine as a chromogen. Finally, sections were counterstained with hematoxylin and mounted. The sections were observed and photographed under the light microscope with the magnification $\times 100$ (Olympus BX 51, Tokyo, Japan). Negative controls had the primary antibody omitted or replaced by non-immune as the primary antibodies.

\section{Statistical Analysis}

SPSS 24.0 software (IBM) was used for data analysis. Measurements were obtained in at least three independent experiments, and were expressed as mean $\pm \mathrm{SD}$. One way ANOVA was carried out for the comparison among the three groups. The Fisher's Least Significant Difference (LSD) test was applied for post-hoc comparisons. A twotailed P-value less than 0.05 was considered to be statistically significant.

\section{Results \\ GBE Improves Depressive Behaviors, Reduces Hippocampus 5-HT And Inhibits Inflammation In HF Mice}

SPT and OFT are two widely used behavior tests used to describe exploratory activities and depressive symptoms. After 4 weeks' treatment, HF mice showed significantly decreased sweet water consumption and grid crossing times. These depressive behaviors were completely reversed by the administration of GBE (Figure 1).

Considering the great impact of 5-HT during the development of depression, we then measured the concentrations of 5 HT in two encephalic regions that play vital roles in depressive behaviors: the hippocampus and cerebral cortex. Interestingly, instead of having lower levels of 5-HT, HF mice had significantly elevated 5-HT concentrations in both cerebral cortex $(176.26 \pm 7.31$ vs $252.28 \pm 3.91 \mathrm{pg} / \mathrm{mg})$ and hippocampus (177.06 \pm 2.10 vs $246.72 \pm 5.25 \mathrm{pg} / \mathrm{mg}$ ) (Figure 2A and B). As a major type of 5-HT receptor localized on the postsynaptic membrane, the $5-\mathrm{HT}_{2 \mathrm{~A}} \mathrm{R}$ mediates calcium influx and subsequent signal transduction. We found that expression of 5$\mathrm{HT}_{2 \mathrm{~A}} \mathrm{R}$, correlated with levels of 5-HT, and were also increased in HF mice (Figure 2C and D). GBE treatment was able to reduce the levels of 5-HT in the hippocampus (162.24 \pm 5.82 vs $246.72 \pm 5.25 \mathrm{pg} / \mathrm{mg}$ ), but GBE did not contribute to significant changes in the level of 5-HT in the cortex, or 5$\mathrm{HT}_{2 \mathrm{~A}} \mathrm{R}$ in either cortex or hippocampus (Figure $2 \mathrm{~B}$ and $\mathrm{C}$ ). 
A Sucrose water test

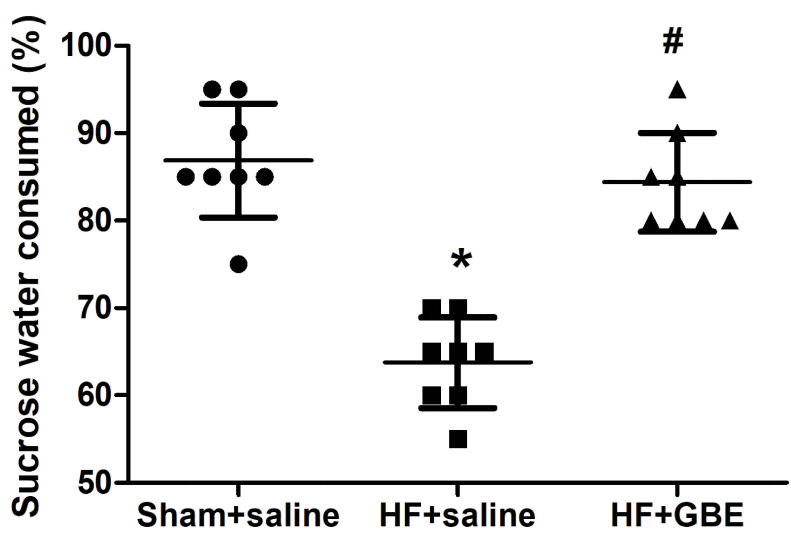

B

Open-field test

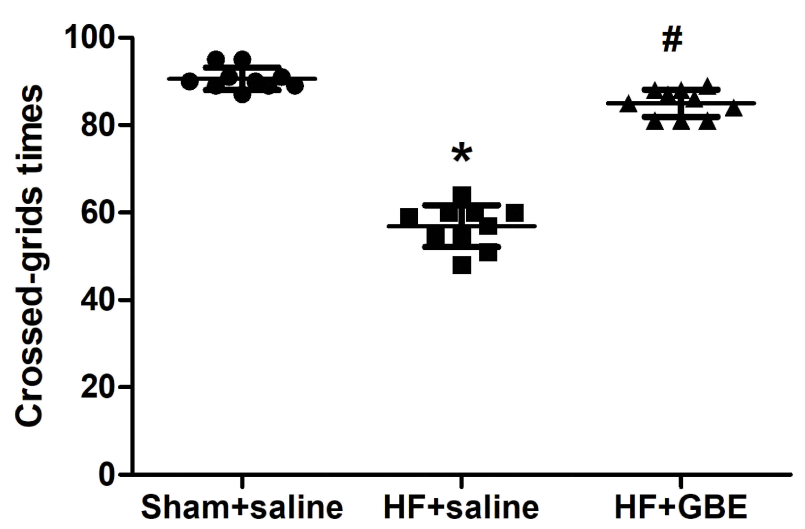

Figure I The results of depressive behaviors. (A) The results of source water test; (B) the results of open-field test. $* \mathrm{P}<0.05$ comparing with sham+saline group. ${ }^{\# P<0.05}$ comparing with $\mathrm{HF}+\mathrm{GBE}$ group.
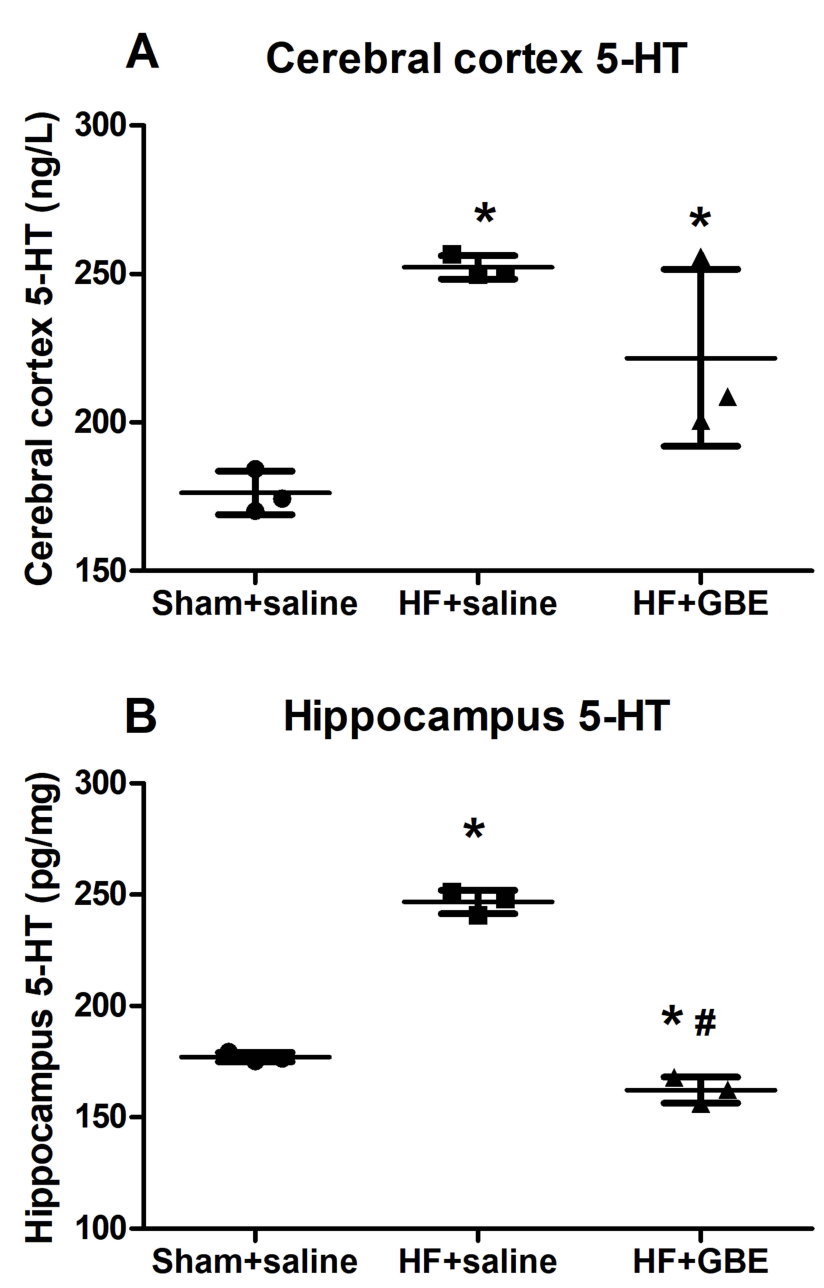
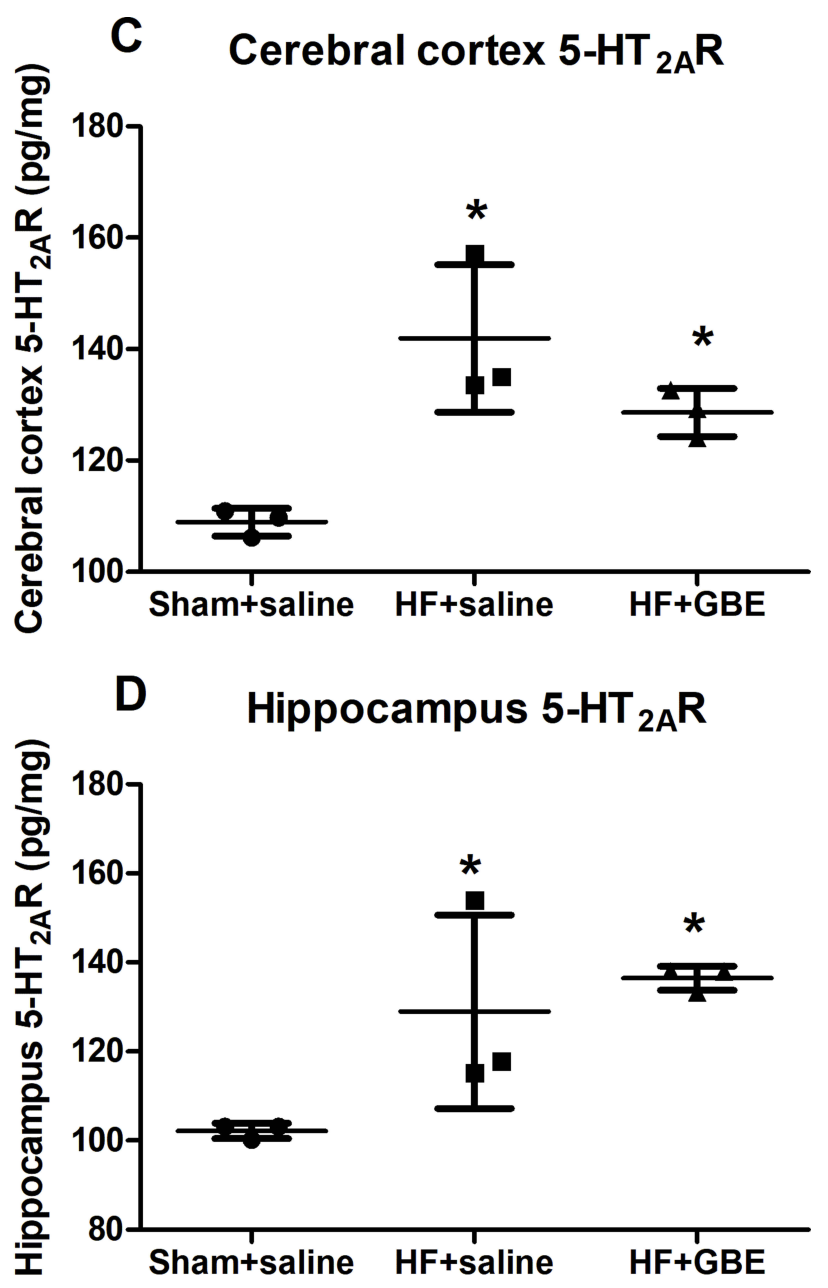

Figure 2 The concentration of 5- $\mathrm{HT}$ and 5- $\mathrm{HT}_{2 \mathrm{~A}} \mathrm{R}$ in cerebral cortex and hippocampus. (A) The level of 5-HT in cerebral cortex. (B) The level of 5- $\mathrm{HT}$ in hippocampus. (C) The level of $5-\mathrm{HT}_{2 \mathrm{~A}} \mathrm{R}$ in cerebral cortex. (D) The level of $5-\mathrm{HT}_{2 \mathrm{~A}} \mathrm{R}$ in hippocampus. All the proteins were detected by ELISA. *P<0.05 comparing with sham+saline group. ${ }^{\#} \mathrm{P}<0.05$ comparing with $\mathrm{HF}+\mathrm{GBE}$ group.

As strong links between inflammatory and serotonergic responses were reported before, we further explored levels of pro-inflammatory cytokines in the hippocampus where the levels of 5-HT were significantly different between the 
three groups. Both Western blot and immunohistochemistry showed that mice with HF had increased expression of both TNF- $\alpha$ and IL- $1 \beta$ while the use of GBE significantly prevented this change (Figure $3 \mathrm{~A}$ and $\mathrm{B}$ ).

\section{GBE Improves Cardiac Performance, Promotes Anti-Apoptotic Responses And Blocks 5-HT Release From Platelets}

As shown in Figure 4, HF mice exhibited decreased LVEF values, increased levels of serum BNP and worsened myocardial fibrosis. GBE treatment not only improved echocardiographic performance, but also reduced the scar size and collagen deposition. In addition, the myocardial fibrosis area decreased from $15.50 \pm 1.73 \%$ in the $\mathrm{HF}+$ saline group to $8.25 \pm 1.71 \%$ in the $\mathrm{HF}+\mathrm{GBE}$ group.

The therapeutic effects of GBE were accompanied by decreased levels of cleaved caspase-3. The anti-apoptotic function of GBE was also confirmed by the decline of Bax and elevation of Bcl-2 expression (Figure 5A). Further analysis indicated that HF mice receiving GBE treatment, compared with mice in the sham and HF saline groups, had similar levels of VEGF but significantly higher levels of HIF-1 (Figure 5B). Moreover, promoted 5-HT release into the plasma was identified in HF mice, which is characterized by increased levels of 5-HT in the serum $(144.97 \pm 4.73$ vs $206.63 \pm 26.22 \mathrm{ug} / \mathrm{L})$ but decreased levels of 5 -HT in the platelets $(189.85 \pm 19.78$ vs $160.82 \pm 20.73 \mathrm{ug} / \mathrm{L})$ (Figure $5 \mathrm{C}$ and D). This process was blocked by GBE administration, and potential mechanisms may involve the up-regulation of the sole 5-HT receptor in the platelets, $5-\mathrm{HT}_{2 \mathrm{~A}} \mathrm{R}(102.59$ \pm 10.73 vs $127.08 \pm 14.46 \mathrm{ug} / \mathrm{L}$ ) (Figure $5 \mathrm{E}$ ).

\section{Discussion}

Recently, depression has shown an increasing prevalence in patients with HF and contributed to elevated mortality. ${ }^{3-5}$ This may be caused by the fact that pathogenesis of depression and HF share similar molecular mechanisms, including the activation of the HPA axis ${ }^{19,20}$ and inflammation. ${ }^{21,22}$ However, it is noteworthy that treatment with antidepressants, such as selective serotonin reuptake inhibitors (SSRI) and monoamine oxidase inhibitors (MOI), is not effective in

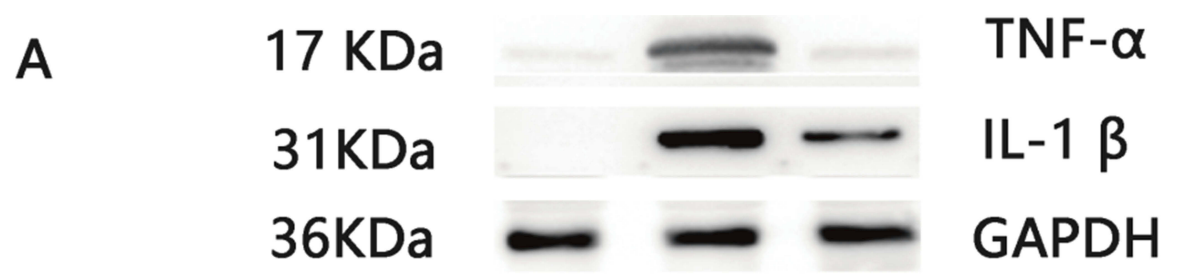

B

Sham+saline $\mathrm{HF}+$ saline $\mathrm{HF}+\mathrm{GBE}$

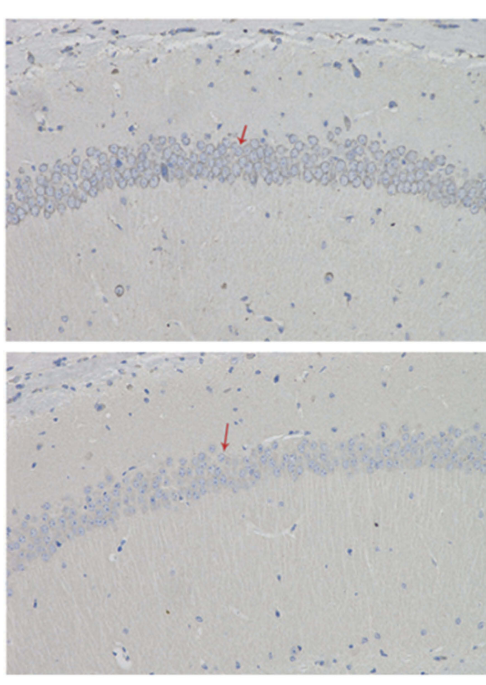

Sham+saline

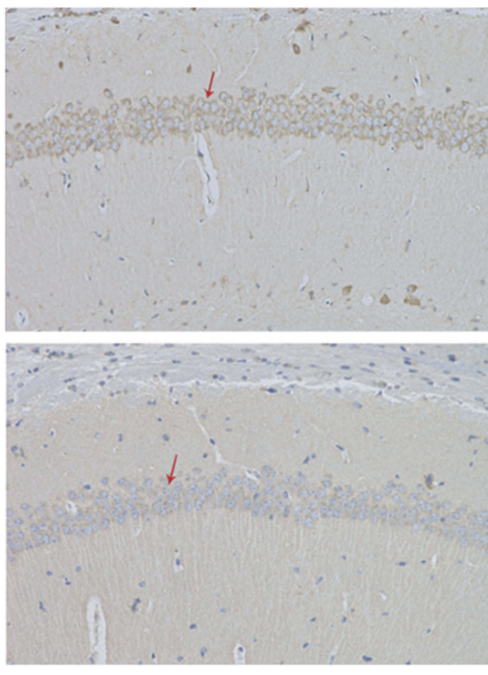

$\mathrm{HF}+$ saline

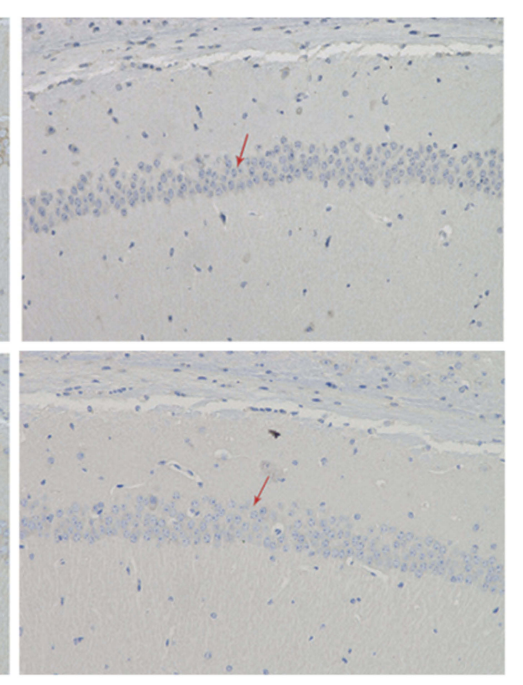

IL_1 $\beta$

TNF- $\alpha$

Figure 3 The response of inflammation. (A) The concentration of TNF-aand IL- $\boldsymbol{\beta} \boldsymbol{\beta}$ in hippocampus detected by WB, and GAPDH as loading control. (B) The expression of TNF- $\alpha$ and IL-I $\boldsymbol{\beta}$ in hippocampus by immunohistochemistry, and the magnification was 100 times the original size. Red arrows indicate examples of the expression of related cytokines. 


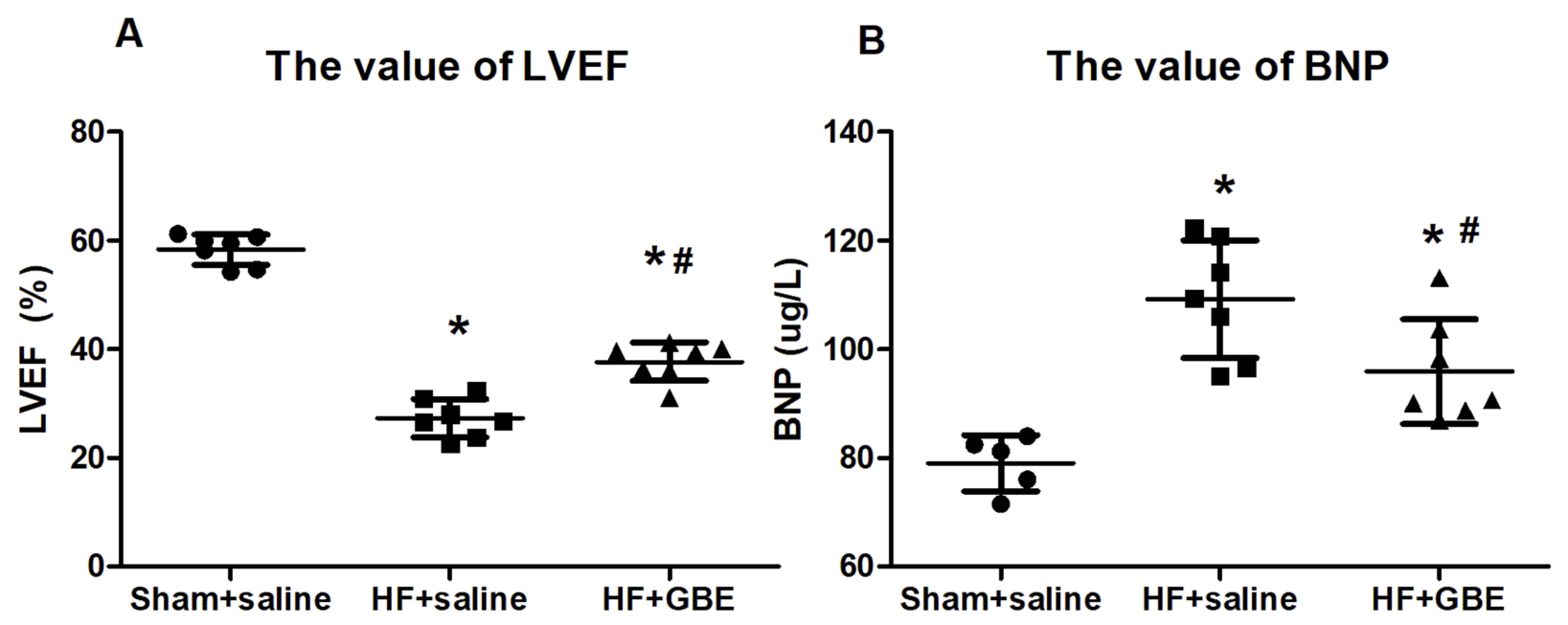

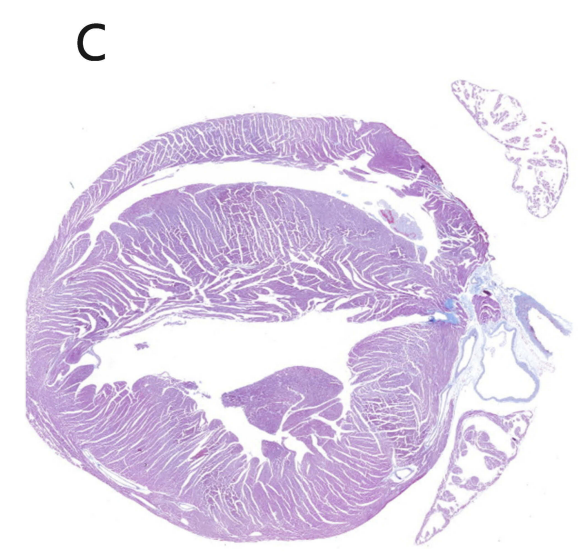

Sham + saline
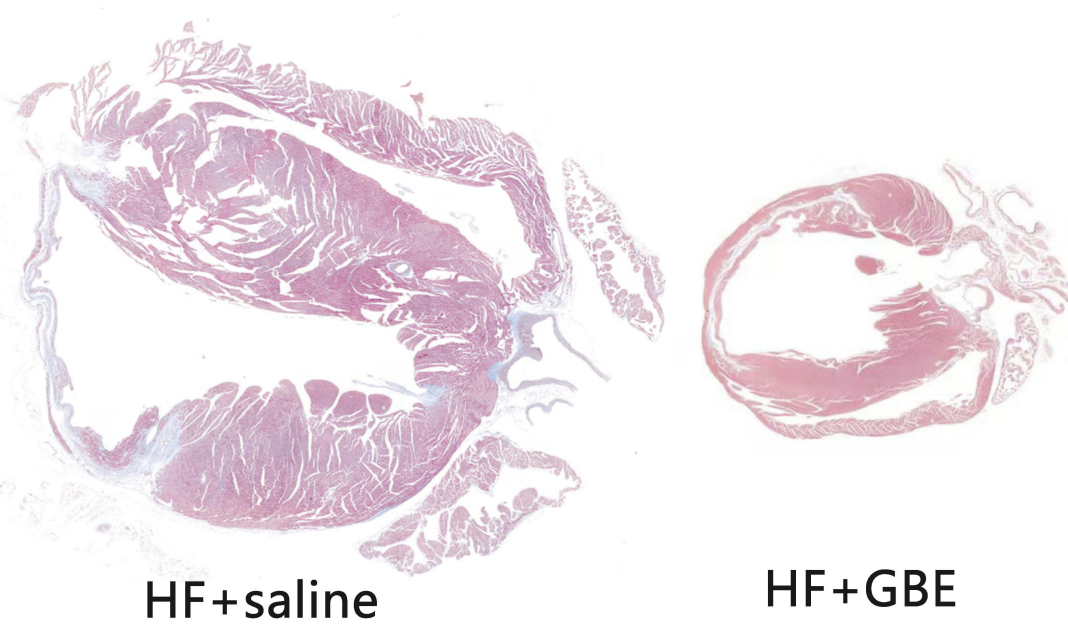

Figure 4 The cardiac function. (A) The value of LVEF by echocardiography. (B) The level of BNP by ELISA. (C) The myocardial section in Masson's staining, and the magnification was 5 times the original size. ${ }^{*} \mathrm{P}<0.05$ comparing with sham+saline group. ${ }^{\#} \mathrm{P}<0.05$ comparing with $\mathrm{HF}+\mathrm{GBE}$ group.

improving the behavioral and cardiovascular outcomes of HF patients. ${ }^{23}$ What is worse, the side effects of antidepressants may even increase the all-cause and cardiovascular mortality in HF patients, irrespective of depression. ${ }^{24}$ Therefore, the need to develop effective pharmaceuticals for HF patients with depression is urgent.

In the present study, we investigate the therapeutic effects of GBE in a depressive HF mouse model. Our results confirmed that almost all mice with an LVEF of less than $30 \%$ developed depressive behaviors characterized by reduced sweet water consumption and decreased exploratory actions (Figure 1). Therefore, this model can be used effectively and reliably in screening for novel drugs which can treat both depression and HF simultaneously. 5-HT, as a neurotransmitter, has been widely considered to play important roles in the process of depression. Surprisingly, instead of having lower levels of central 5-HT, HF mice showed increased 5-HT in both the cortex and hippocampus (Figure 2). To our knowledge, the relationship between heart failure and cortex and hippocampus 5-HT has not been elucidated. We have such hypotheses: 1) the results are different from regular discovery that depression is connected with lower 5-HT in synaptic cleft. Because we detected the whole cortex and hippocampus homogenate rather than synaptic cleft. There might be an amount of 5HT stored in the vesicles of the neurons. 2) Heart failure might play an important role in increasing cortex and hippocampus 5-HT. 3) We previously demonstrated that the hippocampus does not express tryptophan hydroxylase 2 (tph2), which is responsible for 5-HT biosynthesis in the brain. ${ }^{8}$ Therefore, higher 5-HT levels in the hippocampus can only be explained as increased projection of 5-HT from other areas, such as the cerebral cortex. Meanwhile, the increased expression of $5-\mathrm{HT}_{2 \mathrm{~A}} \mathrm{R}$ identified in the cortex 


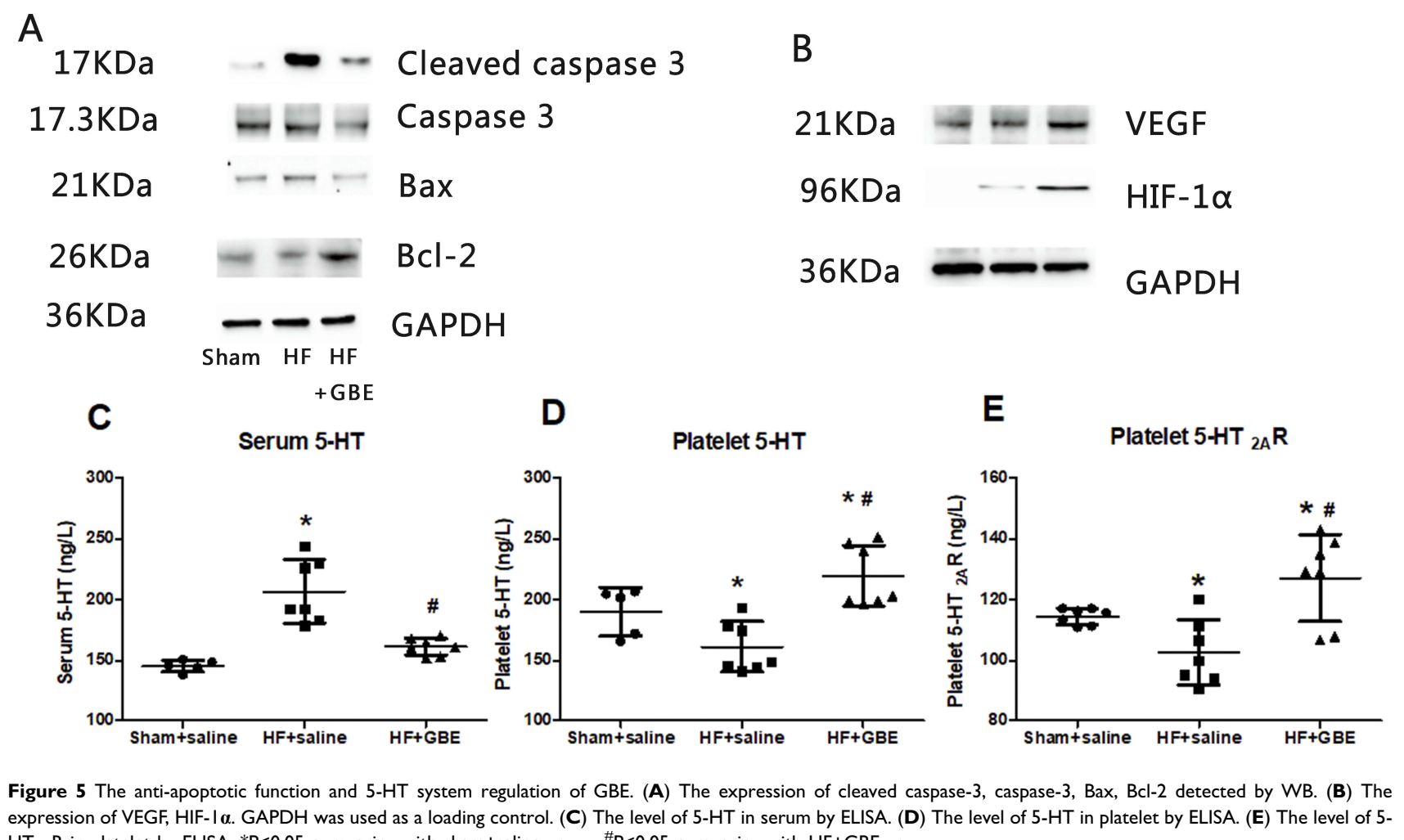

expression of VEGF, HIF- $\mid \boldsymbol{\alpha}$. GAPDH was used as a loading control. (C) The level of 5 -HT in serum by ELISA. (D) The
$\mathrm{HT}_{2 \mathrm{~A}} \mathrm{R}$ in platelet by ELISA. ${ }^{*} \mathrm{P}<0.05$ comparing with sham+saline group. ${ }^{*} \mathrm{P}<0.05$ comparing with HF+GBE group.

and hippocampus in HF mice (Figure 2) always indicates promoted 5-HT reuptake from the synaptic clefts. ${ }^{25,26} 4$ ) It has also been reported that HF, which normally results in hypoxia, significantly contributes to structural and functional changes of the cerebral cortex and hippocampus in humans and rodents. ${ }^{27,28} 5$ ) In addition, dysfunctions in the central serotonergic system, such as up-regulation of 5 $\mathrm{HT}_{2 \mathrm{~A}} \mathrm{R}$, may further lead to worsened prognosis in $\mathrm{HF}$ through enhanced HPA axis activity.

While GBE could not reverse the elevation of 5-HT in cortex and 5- $\mathrm{HT}_{2 \mathrm{~A}} \mathrm{R}$ in cortex and hippocampus, we hypothesis that GBE improved the depressive behaviors of HF mice via decreasing 5-HT and inflammation in hippocampus, rather than reducing the function of $5-\mathrm{HT}_{2 \mathrm{~A}} \mathrm{R}$. Since 5-HT may also link to the immune system, ${ }^{29}$ we then tested levels of TNF- $\alpha$ and IL1- $\beta$, which are two major cytokines participating in neural inflammation during the process of depression. ${ }^{30,31}$ Although we did observe significantly increased expression of TNF- $\alpha$ and IL1- $\beta$ in the hippocampus, where levels of 5-HT were also elevated, it is still in doubt whether these cytokines were secreted by regional microglia or transported through blood-brain barrier (BBB) from the peripheral blood.

Consistent with a previous clinical study, ${ }^{32}$ we found that mice with HF showed significantly increased serum levels of 5-HT with a reduction in platelet 5-HT storage (Figure 5), indicating heightened release of 5-HT from the platelets. In addition to a neurotransmitter, 5-HT also functions as a potent vasoconstrictor or a vasodilator depending on the integrity of the vessel endothelium. Released 5-HT from the platelets, in combination with ADP and other substances, can also mobilize the coagulation system and then promote blood clotting. Besides, increased 5-HT may further trigger the renin-angiotensin-aldosterone system (RAAS), which regulates the release of vasopressin and norepinephrine. ${ }^{33}$ The raised levels of vasopressin could cause peripheral vasoconstriction and result in higher blood pressure and increased sympathetic activities, which both promote deterioration into heart failure. On the other hand, elevated levels of norepinephrine lead to increased cardiac contractility and heart rate which can initially help maintaining cardiac output, but be deleterious in the long term.

GBE is a widely used natural product in clinical practice in several countries, including Germany, the USA and Japan. It has been shown to be effective in treating multiple diseases. ${ }^{13-16}$ However, inconsistent results were generated by research groups as a result of different manufacturing standards adopted by pharmaceutical companies. ${ }^{34,35}$ Therefore, the efficacy and underlying mechanisms of 
GBE action remain largely obscure. GBE utilized in this research was extracted according to the criterion of Chinese pharmacopoeia. Cao et al had reported a qualitative analysis to establish the ingredients of GBE (dripping pills or Yinxingye Diwan in Chinese) based on high-resolution mass spectrometry and metabolomics technology and they found higher level of flavonols in GBE. ${ }^{36}$ The main ingredients of GBE contain ginkgolide A, B, C, etc., and GBE has been proved to have antidepressant-like effect on animals with depressive behaviors. And the mechanism of GBE in relieving depressive behaviors is related with reducing inflammatory response which is involved in the development of depression. ${ }^{37}$

In consistent with former studies, we focused on hippocampus for the following reasons: firstly, research have demonstrated that heart failure, low cardiac output, a generalized hypoxia statue, ischemia and chronic stress would contribute to cerebral cortex and hippocampus change. Secondly, in our previous experiment, we discovered that 5-HT content in nerve fibers projected to the hippocampus was increased during cardiac failure. Thirdly, Ginkgo biloba extract has been proved to alter $\mathrm{Bax}$ to $\mathrm{Bcl}-2$ expression ratio in the hippocampus of senescence accelerated mice. ${ }^{38}$ In the present paper, we confirm that GBE has anti-inflammatory effects in the hippocampus (Figure 3) and is able to rescue enhanced projection of 5-HT to the hippocampus (Figure 2). Its regulatory effects in neural immune and 5-HT systems may be the main mechanisms of relieving depressive actions observed in our behavior tests (Figure 1). Moreover, we successfully show that this particular pharmaceutical product shares similar therapeutic effects in HF mice compared with other Ginkgo biloba products (Figure 4). ${ }^{39,40}$ Further analysis reveals that its cardioprotective outcomes are associated with reduced apoptotic signals (Figure 5). Apoptosis is actively involved in the development of HF featured by increased cleaved caspase-3, increased Bcl-2 and decreased Bax. ${ }^{41-43}$ Anti-apoptotic properties can be achieved by activation of endogenous regenerative mechanisms, such as VEGF and HIF-1 signaling pathways. ${ }^{44-46}$ By exploring the expression of VEGF and HIF-1 in the myocardium, we suggest that apoptosis suppression under the administration of GBE is dependent on HIF-1, but not VEGF-related pathways. GBE also exhibited negative regulatory effects on 5-HT levels in the serum, probably via promoting $5-\mathrm{HT}_{2 \mathrm{~A}} \mathrm{R}$ mediated 5-HT uptake in the platelets. This may particularly contribute to the beneficial effects of GBE in patients with $\mathrm{HF}$ as platelet aggravation is a major risk factor for HF and is believed to be associated with poor prognosis in HF patients. ${ }^{47,48}$

\section{Conclusion}

Overall, this experimental study provides the first evidence that GBE rescues depressive behaviors and cardiac failure simultaneously in a depressive HF mouse model. Potential mechanisms may include the down-regulation of hippocampus 5-HT, inhibition of neural inflammation, blockage of 5-HT release from platelets and activation of HIF-1 mediated antiapoptosis. This research also provides some novel insights in the area of psychocardiology. Firstly, HF is accompanied by depressive symptoms in all subjects involved in the experiments. This parallels the comorbidity of HF and depression we face in clinical practice. Besides, we raise the possibility of utilizing the 5-HT system as a therapeutic target in patients with HF and depression. However, it should be remembered that further research is still needed to explain the source of increased 5-HT and pro-inflammatory factors in the hippocampus. Moreover, it is also important to understand whether and how decreased 5-HT in the blood finally results in suppression of apoptosis.

\section{Acknowledgment}

This work was supported by the National Key Basic Research Program of China (973 program) grant no. 2015 CB554402.

\section{Disclosure}

The authors report no conflicts of interest in this work.

\section{References}

1. Vos T, Abajobir AA, Abate KH, et al. Global, regional, and national incidence, prevalence, and years lived with disability for 328 diseases and injuries for 195 countries, 1990-2016: a systematic analysis for the global burden of disease study 2016. Lancet (London, England). 2017;390(10100):1211-1259. doi:10.1016/S0140-6736(17)32154-2

2. Benjamin EJ, Blaha MJ, Chiuve SE, et al. Heart disease and stroke statistics-2017 update: a report from the American Heart Association. Circulation. 2017;135(10):e146-e603.

3. Angermann CE, Gelbrich G, Stork S, et al. Somatic correlates of comorbid major depression in patients with systolic heart failure. Int J Cardiol. 2011;147(1):66-73. doi:10.1016/j.ijcard.2009.07.044

4. Faller H, Stork S, Schuler M, et al. Depression and disease severity as predictors of health-related quality of life in patients with chronic heart failure-a structural equation modeling approach. J Card Fail. 2009;15 (4):286-292.e282. doi:10.1016/j.cardfail.2008.10.022

5. Husain MI, Chaudhry IB, Husain MO, et al. Depression and congestive heart failure: a large prospective cohort study from Pakistan. $J$ Psychosom Res. 2019;120:46-52. doi:10.1016/j.jpsychores.2019.03.008

6. Su J, Wang J, Ma Y, et al. Inflammation associated with chronic heart failure leads to enhanced susceptibility to depression. FEBS J. 2019. doi:10.1111/febs. 14839

7. Frey A, Popp S, Post A, et al. Experimental heart failure causes depression-like behavior together with differential regulation of inflammatory and structural genes in the brain. Front Behav Neurosci. 2014;8:376. doi:10.3389/fnbeh.2014.00376 
8. Liu M, Liu J, Zhang L, Geng Q, Ge Y. Antidepressant-like effects of ginseng fruit saponin in myocardial infarction mice. Biomed Pharmacother. 2019;115:108900. doi:10.1016/j.biopha.2019.108900

9. Jokinen J, Nordstrom P. HPA axis hyperactivity and cardiovascular mortality in mood disorder inpatients. J Affect Disord. 2009;116(12):88-92. doi:10.1016/j.jad.2008.10.025

10. Majidi J, Kosari-Nasab M, Salari AA. Developmental minocycline treatment reverses the effects of neonatal immune activation on anxiety- and depression-like behaviors, hippocampal inflammation, and HPA axis activity in adult mice. Brain Res Bull. 2016;120:1-13. doi:10.1016/j.brainresbull.2015.10.009

11. Hemrick-Luecke SK, Evans DC. Comparison of the potency of MDL 100,907 and SB 242084 in blocking the serotonin $(5-\mathrm{HT})(2)$ receptor agonist-induced increases in rat serum corticosterone concentrations: evidence for 5-HT(2A) receptor mediation of the HPA axis. Neuropharmacology. 2002;42(2):162-169. doi:10.1016/S0028-3908(01) 00166-6

12. Vogelzangs N, Beekman AT, Milaneschi Y, Bandinelli S, Ferrucci L, Penninx BW. Urinary cortisol and six-year risk of all-cause and cardiovascular mortality. J Clin Endocrinol Metab. 2010;95 (11):4959-4964. doi:10.1210/jc.2010-0192

13. Sun T, Wang X, Xu H. Ginkgo biloba extract for angina pectoris: a systematic review. Chin J Integr Med. 2015;21(7):542-550. doi:10.1007/s11655-015-2070-0

14. Kang JM, Lin S. Ginkgo biloba and its potential role in glaucoma Curr Opin Ophthalmol. 2018;29(2):116-120. doi:10.1097/ICU.0000 000000000459

15. Tulsulkar J, Glueck B, Hinds TD Jr, Shah ZA. Ginkgo biloba extract prevents female mice from ischemic brain damage and the mechanism is independent of the HO1/Wnt pathway. Transl Stroke Res. 2016;7(2):120-131. doi:10.1007/s12975-015-0433-7

16. Dai CX, Hu CC, Shang YS, Xie J. Role of Ginkgo biloba extract as an adjunctive treatment of elderly patients with depression and on the expression of serum S100B. Medicine. 2018;97(39):e12421. doi:10.1097/ MD.0000000000012421

17. Diamond BJ, Bailey MR. Ginkgo biloba: indications, mechanisms, and safety. Psychiatr Clin North Am. 2013;36(1):73-83. doi:10.1016/ j.psc.2012.12.006

18. Liu L, Dong Y, Shan X, Li L, Xia B, Wang H. Anti-depressive effectiveness of baicalin in vitro and in vivo. Molecules. 2019;24 (2):326. doi:10.3390/molecules24020326

19. Sivukhina EV, Poskrebysheva AS, Smurova IV, et al. Altered hypothalamic-pituitary-adrenal axis activity in patients with chronic heart failure. Horm Metab Res. 2009;41(10):778-784. doi:10.1055/s0029-1224182

20. Juruena MF, Bocharova M, Agustini B, Young AH. Atypical depression and non-atypical depression: is HPA axis function a biomarker? A systematic review. J Affect Disord. 2018;233:45-67. doi:10.1016/j. jad.2017.09.052

21. Miller AH, Raison CL. The role of inflammation in depression: from evolutionary imperative to modern treatment target. Nat Rev Immunol. 2016;16(1):22-34. doi:10.1038/nri.2015.5

22. Ter Maaten JM, Damman K, Verhaar MC, et al. Connecting heart failure with preserved ejection fraction and renal dysfunction: the role of endothelial dysfunction and inflammation. Eur J Heart Fail. 2016;18(6):588-598. doi:10.1002/ejhf.2016.18.issue-6

23. Rajeswaran T, Plymen CM, Doherty AM. The effect of antidepressant medications in the management of heart failure on outcomes: mortality, cardiovascular function and depression - a systematic review. Int J Psychiatry Clin Pract. 2018;22(3):164-169. doi:10.10 80/13651501.2017.1401085

24. Brouwers C, Christensen SB, Damen NL, et al. Antidepressant use and risk for mortality in 121,252 heart failure patients with or without a diagnosis of clinical depression. Int J Cardiol. 2016;203:867-873. doi:10.1016/j.ijcard.2015.11.032
25. Hrdina PD, Demeter E, Vu TB, Sotonyi P, Palkovits M. 5-HT uptake sites and 5-HT2 receptors in brain of antidepressant-free suicide victims/ depressives: increase in 5-HT2 sites in cortex and amygdala. Brain Res. 1993;614(1-2):37-44. doi:10.1016/0006-8993(93)91015-K

26. Nichols CD. Serotonin 5-HT(2A) receptor function as a contributing factor to both neuropsychiatric and cardiovascular diseases. Cardiovasc Psychiatry Neurol. 2009;2009:475108. doi:10.1155/2009/475108

27. Suzuki H, Sumiyoshi A, Matsumoto Y, et al. Structural abnormality of the hippocampus associated with depressive symptoms in heart failure rats. NeuroImage. 2015;105:84-92. doi:10.1016/j.neuroimage.2014.10.040

28. Suzuki H, Matsumoto Y, Ota H, et al. Hippocampal blood flow abnormality associated with depressive symptoms and cognitive impairment in patients with chronic heart failure. Circ J. 2016;80 (8):1773-1780. doi:10.1253/circj.CJ-16-0367

29. Wu H, Denna TH, Storkersen JN, Gerriets VA. Beyond a neurotransmitter: the role of serotonin in inflammation and immunity. Pharmacol Res. 2019;140:100-114. doi:10.1016/j.phrs.2018.06.015

30. Goshen I, Kreisel T, Ben-Menachem-Zidon O, et al. Brain interleukin-1 mediates chronic stress-induced depression in mice via adrenocortical activation and hippocampal neurogenesis suppression. Mol Psychiatry. 2008;13(7):717-728. doi:10.1038/sj.mp.4002055

31. Li B, Wang B, Chen M, Li G, Fang M, Zhai J. Expression and interaction of TNF-alpha and VEGF in chronic stress-induced depressive rats. Exp Ther Med. 2015;10(3):863-868. doi:10.3892/etm.2015.2641

32. Nigmatullina RR, Kirillova VV, Jourjikiya RK, et al. Disrupted serotonergic and sympathoadrenal systems in patients with chronic heart failure may serve as new therapeutic targets and novel biomarkers to assess severity, progression and response to treatment. Cardiology. 2009;113(4):277-286. doi:10.1159/000205962

33. Ramage AG. Central cardiovascular regulation and 5-hydroxytryptamine receptors. Brain Res Bull. 2001;56(5):425-439. doi:10.1016/ S0361-9230(01)00612-8

34. Mantle D, Wilkins RM, Gok MA. Comparison of antioxidant activity in commercial Ginkgo biloba preparations. J Altern Complement Med. 2003;9(5):625-629. doi:10.1089/107555303322524472

35. Woelkart K, Feizlmayr E, Dittrich P, et al. Pharmacokinetics of bilobalide, ginkgolide A and B after administration of three different Ginkgo biloba L. preparations in humans. Phytother Res. 2010;24 (3):445-450. doi:10.1002/ptr.v24:3

36. Cao Guoxiu LW, Hui Y. Rapid identification of constituents from different Ginkgo biloba preparations by high resolution mass spectrometry and metabolomics technology. J China Pharm Univ. 2018;49 (4):441-448.

37. Liang Z, Bai S, Shen P, et al. GC-MS-based metabolomic study on the antidepressant-like effects of diterpene ginkgolides in mouse hippocampus. Behav Brain Res. 2016;314:116-124. doi:10.1016/j. bbr.2016.08.001

38. Mak YT, Chan WY, Lam WP, Yew DT. Immunohistological evidences of Ginkgo biloba extract altering Bax to Bcl-2 expression ratio in the hippocampus and motor cortex of senescence accelerated mice. Microsc Res Tech. 2006;69(8):601-605. doi:10.1002/(ISSN)1097-0029

39. Panda VS, Naik SR. Cardioprotective activity of Ginkgo biloba phytosomes in isoproterenol-induced myocardial necrosis in rats: a biochemical and histoarchitectural evaluation. Exp Toxicol Pathol. 2008;60(4-5):397-404. doi:10.1016/j.etp.2008.03.010

40. El-Boghdady NA. Increased cardiac endothelin-1 and nitric oxide in adriamycin-induced acute cardiotoxicity: protective effect of Ginkgo biloba extract. Indian J Biochem Biophys. 2013;50(3):202-209.

41. Yang B, Ye D, Wang Y. Caspase-3 as a therapeutic target for heart failure. Expert Opin Ther Targets. 2013;17(3):255-263. doi:10.1517/ 14728222.2013.745513

42. Francis GS, Anwar F, Bank AJ, Kubo SH, Jessurun J. Apoptosis, Bcl2 , and proliferating cell nuclear antigen in the failing human heart: observations made after implantation of left ventricular assist device. $J$ Card Fail. 1999;5(4):308-315. doi:10.1016/S1071-9164(99)91335-0 
43. Moorjani N, Westaby S, Narula J, et al. Effects of left ventricular volume overload on mitochondrial and death-receptor-mediated apoptotic pathways in the transition to heart failure. Am J Cardiol. 2009;103(9):1261-1268. doi:10.1016/j.amjcard.2009.01.013

44. Dias S, Shmelkov SV, Lam G, Rafii S. VEGF(165) promotes survival of leukemic cells by Hsp90-mediated induction of Bcl-2 expression and apoptosis inhibition. Blood. 2002;99(7):2532-2540. doi:10.1182/ blood.V99.7.2532

45. Heider U, Kaiser M, Sterz J, et al. Histone deacetylase inhibitors reduce VEGF production and induce growth suppression and apoptosis in human mantle cell lymphoma. Eur J Haematol. 2006;76 (1):42-50. doi:10.1111/EJH.2006.76.issue-1
46. Fu JD, Yao JJ, Wang H, et al. Effects of EGCG on proliferation and apoptosis of gastric cancer SGC7901 cells via down-regulation of HIF1alpha and VEGF under a hypoxic state. Eur Rev Med Pharmacol Sci. 2019;23(1):155-161. doi:10.26355/eurrev 20190116759

47. de Meirelles LR, Resende Ade C, Matsuura C, et al. Platelet activation, oxidative stress and overexpression of inducible nitric oxide synthase in moderate heart failure. Clin Exp Pharmacol Physiol. 2011;38(10):705-710. doi:10.1111/j.1440-1681.2011.05580.x

48. Kim JH, Shah P, Tantry US, Gurbel PA. Coagulation abnormalities in heart failure: pathophysiology and therapeutic implications. Curr Heart Fail Rep. 2016;13(6):319-328. doi:10.1007/s11897-016-0308-6

\section{Publish your work in this journal}

Neuropsychiatric Disease and Treatment is an international, peerreviewed journal of clinical therapeutics and pharmacology focusing on concise rapid reporting of clinical or pre-clinical studies on a range of neuropsychiatric and neurological disorders. This journal is indexed on PubMed Central, the 'PsycINFO' database and CAS, and is the official journal of The International Neuropsychiatric Association (INA). The manuscript management system is completely online and includes a very quick and fair peer-review system, which is all easy to use. Visit http://www.dovepress.com/testimonials.php to read real quotes from published authors. 